\title{
HOW THE SHIFT TO OMNI-CHANNEL COMMERCE STRATEGY AIDS A FURNITURE RETAILER'S COMPETITIVE DIFFERENTIATION: THE CASE OF XXX LUTZ IN AUSTRIA
}

\author{
DOI: 10.17261/Pressacademia.2019.1131 \\ JMML-V.6-ISS.3-2019(6)-p.197-203
}

Lisa Maria Wieser ${ }^{1}$, Yung-Shen Yen ${ }^{2}$

${ }^{1}$ University of Applied Sciences Upper Austria, Campus Steyr, Austria. lisa.wieser@students.fh-steyr.at, ORCID: 0000-0002-0969-3275

${ }^{2}$ Providence University, Department of Computer Science and Information Management, Taichung, Taiwan. ysyen@pu.edu.tw, ORCID: 0000-0003-1146-9771

Date Received: June 22, 2019

Date Accepted: September 22, 2019

To cite this document

Wieser, L., M., Yen, Y., S., (2019). How the shift to omni-channel commerce strategy aids a furniture retailer's competitive differentiation: the case of xxx Lutz in Austria. Journal of Management, Marketing and Logistics (JMML), V.6(3), p.197-203

Permemant link to this document: http://doi.org/10.17261/Pressacademia.2019.1131

Copyright: Published by PressAcademia and limited licenced re-use rights only.

\section{ABSTRACT}

Purpose- This study aims to devise a well-grounded omni-channel commerce strategy for the furniture retailers.

Methodology- We used XXX Lutz, a well-established furniture retailer in Austria, as a study case. A content analysis of using website information, academic papers and social media documents, was conducted.

Findings- The finding shows that XXX Lutz's shift to an omni-channel commerce strategy requires a smoother integration of all utilized channels. This could be achieved by two approaches, namely informational integration and physical integration. Moreover, potential challenges XXX Lutz might face by adopting an omni-channel strategy are identified and adequate solutions are proposed.

Conclusion- This study extends the understanding of the omni-channel strategy for the furniture retailers. Our findings affirm the assumption that informational and physical integrations are two important dimensions of channel integration for the omni-channel retailers.

Keywords: Omni-channel, cross-channel, informational integration, physical integration, retailing.

JEL Codes: M30, M31

\section{INTRODUCTION}

The emergence of the World Wide Web, technological advancement as well as increasing usage of mobile devices have tremendously changed the shopping behavior of customers (Cochoy, 2012). Along with the increasing fusion of sales channels, this changing consumer behavior is one of the main reasons for the modified retail landscape. Nowadays, consumers are ubiquitously connected and present on multiple channels (Schmieder, 2010). In the 21st century, consumers look for information online, gather existing customer feedback from social media platforms and might finally decide to purchase a product from a physical store (Salma and Anil, 2015). This calls firms and retailers to rethink their current handling of sales channels for the sake of not losing customers in the close future and successfully competing with rivals (Schmieder, 2010), the Austrian furniture retailer XXX Lutz alike.

Considering that just recently one of XXX Lutz main competitors, the Swedish furniture retailer IKEA, has successfully introduced an omni-channel commerce strategy using a virtual reality application so that customers can preview IKEA's furniture in their own home (Gist, 2019), highlights the importance of omni-channel commerce in the furniture retail industry. But what exactly is an omni-channel commerce strategy and why should XXX Lutz care? Omni-channel retailing is defined as a strategy that enables clients a seamless shopping experience by unifying a brand's sales channels through transactional as well as informational touchpoints (Beck and Rygl, 2015). Consequently, omni-channel retailing combines the information-richness of online shopping with the personal shopping experience in a physical store (Rigby, 2011). Although with its positioning IKEA targets a different audience than XXX Lutz, this movement must not be ignored, especially since XXX Lutz's customers who pay a premium price will very likely expect a seamless shopping experience in the near future as well. 
Accordingly, this study aims to develop an omni-channel commerce strategy suggestion for the Austrian furniture retailer XXX Lutz and highlights the benefits for both the consumer and the retailer itself. A content analysis was conducted. We used secondary data sources, such as website information, academic papers and social media documents to underline the contents theoretically. Thus, the structure of the study is as follows: After an initial introduction of XXX Lutz, the firm's current sales channel strategy will be analyzed. Subsequently, a well-grounded omni-channel commerce strategy for XXX Lutz will be proposed and benefits for both the consumer and the firm highlighted. After that, this study elaborates on inescapable challenges an omni-channel strategy implementation may carry along and proposes solutions. Finally, the last section concludes this study and briefly discusses both its academic contribution and limitations.

\section{ABOUT THE XXX LUZE GROUP}

In 1945, Gertrude Lutz founded the company "Lutz" in Haag am Hausruck - Austria. Her vision was to satisfy customers through a well sorted, appealing, high-quality product range. At first, Lutz focused on Austrian craftsmanship: The initial products at that time were hand-painted wooden boxes and traditional, country-style furniture. In the following decades, the small and regional furniture shop has gradually turned into one of the largest furniture retailers in the world. The goal was clear: Success through expansion - Lutz became XXX Lutz. The company defines its mission and vision as follows: XXX Lutz is a customer-focused business committed to becoming the best home furnishing retailer in our markets. By setting up clear and strong brand images, XXX Lutz aims to be the number one retailer by providing an outstanding customer experience throughout a great range of products, fair value, and excellent service. The XXX Lutz Group (2019) is proud of its 22.200 employees and has achieved a turnover of 4.4 billion Euro in 2019. Moreover, it is indispensable to mention that the XXX Lutz Group can be divided into three sub-groups: XXX Lutz, Möbelix and Mömax.

Figure 1 depicts the difference between the three sub-groups and the XXX Lutz Group's main competitors. It visualizes a comparison of selected furnishing retailers' strategic positioning according to price and quality. In particular, XXX Lutz offers high-quality products as justification for high prices. To put it in the words of Michael Porter, XXX Lutz and Leiner are pursuing a differentiation strategy while the other big players such as IKEA, Mömax, and Möbelix try to be leaders in costs. However, one must keep in mind that XXX Lutz, Mömax and Möbelix all belong to the same group and only vary in their strategic positioning aiming to appeal to different target customer segments.

Figure 1: Strategic Positioning of Selected Furniture Retailers

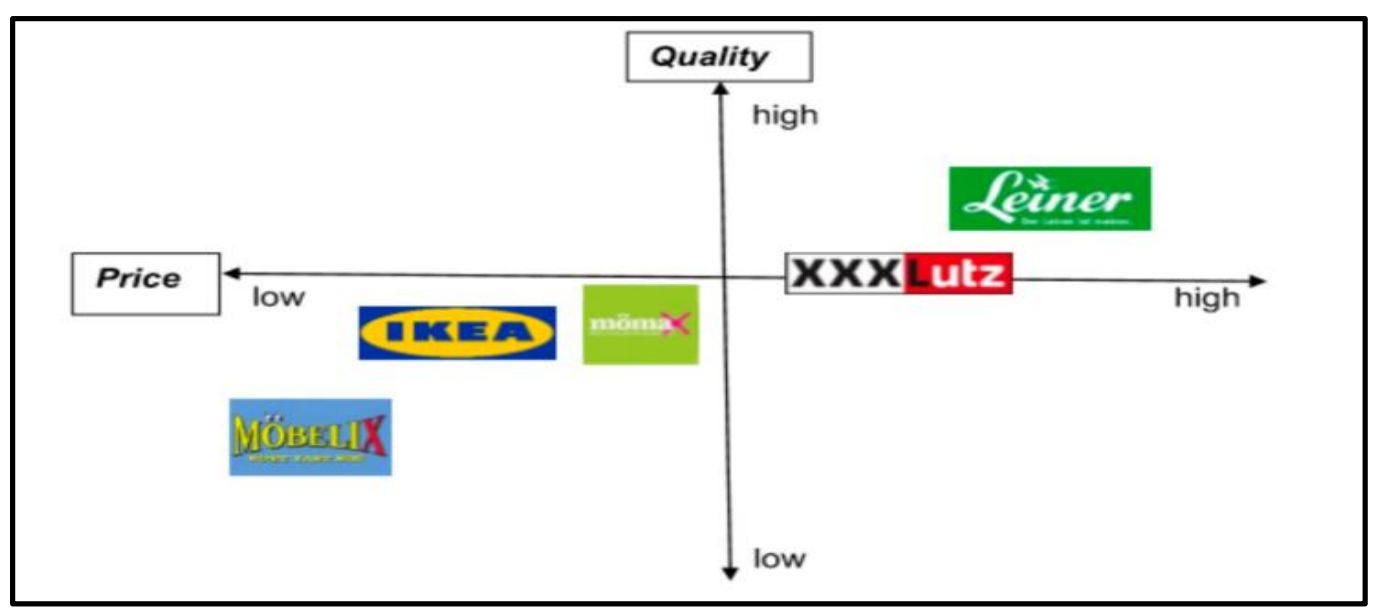

\section{ANALYSIS OF XXX LUTZ'S CURRENT CHANNEL MANAGEMENT SYSTEM}

This section is dedicated to describe XXX Lutz's current channel services. Neslin et al. (2006) indicated that a channel refers to an interaction medium between an organization and its customer. In the case of XXX Lutz, five major channels are in use for information-, communication-, and sales- purposes. These are the firm's physical stores, catalogues and newsletters, websites, mobile applications, and social media platforms.

XXX Lutz primary sales channel is its physical store. Since 1973 the firm has opened an average of 6 stores a year. Nowadays, the firm is with 260 stores in 13 European countries, a well-established furniture retailer (XXX Lutz Group, 2019). XXX Lutz's sales forces are paid on a commission basis. Also, catalogues and newsletters are in use, but solely for information and communication purposes. In other words, the firm does not sell its products and services through this channel. Likewise, the firm accepts customer calls, but by looking on their website where XXX Lutz gives customers the possibility to leave a message, one can clearly feel that the firm intends to shift customer-related communication to its online channels. To put it in another way, the phone is a diminishing communication channel. 
Online channels such as websites, mobile applications, and social media platforms are becoming more and more important in the retail industry. XXX Lutz (2019) has adapted to this trend and offers more than 45.000 furniture products in its online store. Additionally, the firm offers special "online only" offers, excellent online consultation from store experts as well as the possibility to choose a variety of service offers from delivery to assembly. Apart from its website, XXX Lutz launched a mobile application as an additional sales channel. The firm's application increases the accessibility for clients and allows them to purchase anywhere provided that their mobile device is connected to the Internet. Moreover, the firm uses social media platforms, such as Facebook, Instagram, and Linkedln. However, a direct purchase via social media is not possible, but customers wanting to purchase a product they like via social media will be redirected to XXX Lutz's online store.

Due to the fact that XXX Lutz's channels are partially integrated from both the customer and the company's point of view, it can be classified as a cross-channel retailer (Beck and Rygl, 2015). More precisely, as shown in Figure 2, XXX Lutz channels include its physical stores, the firm's catalogues and newsletters, the XXX Lutz website and mobile applications as well as social media platforms. These channels are already partially linked through five main services. First, personal customer accounts ensure customer accessibility through both the retailer's online website, but also through its mobile application. Thus, two channels are used for this service: the retailer's online website as well as its mobile application. Second, the firm has already set measures to connect its online store more closely to the physical store by introducing the "XXX Lutz Dream Space Planner Section". This allows customers to request an appointment in one of XXX Lutz's physical stores during which experts will plan your personal dream space for free. Thus, the main channel used for XXX Lutz's Dream Space Planner Service are the firm's physical stores. Third, the company has already partially implemented QR-Codes, but only for selected products of the retailer's catalogues. Thus, the main channel used is XXX Lutz's catalogues. Fourth, XXX Lutz offers Click \& Collect services to customers that interlink its online website and mobile application with its physical store. Fifth, all of XXX Lutz's data is simultaneously updated on all electronic devices, indicating that its website is the primarily used channel.

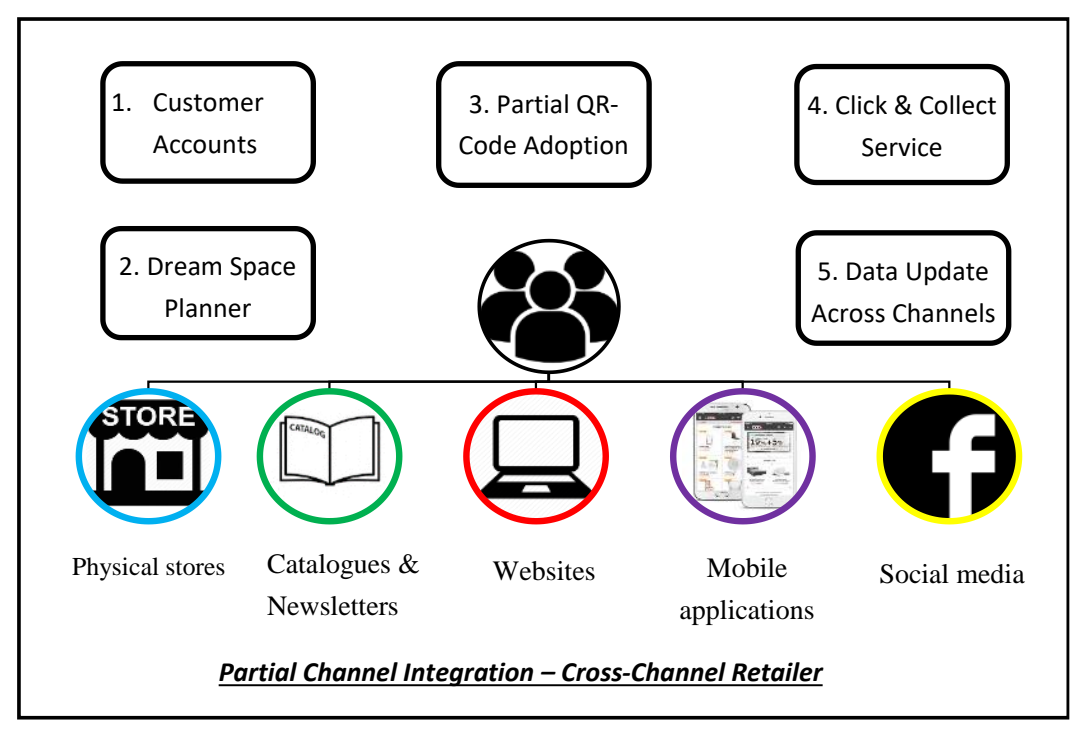

Figure 2. Analysis of XXX Lutz's current channel services offered to clients

\section{PROPOSED OMNI-CHANNEL STRATEGY}

Omni-channel Strategy refers to the strategy regarding the management of diverse channels to offer shoppers a seamless experience across all of the firm's channels (Chatterjee, 2010). In the literature, there are no consensus of the definition of channel integration. Bendoly et al. (2005) demonstrated that channel integration has two factors, namely informational integration and physical integration. Lee and Kim (2010) argued that channel integration has five factors, including information consistency, freedom in channel selection, e-mail marketing, channel reciprocity, and customer service. Frasquet and Miquel (2017) found that channel integration has two factors, namely reciprocity and coordination. Reciprocity refers to the possibility of crossing the channels while shopping, and coordination refers to the alignment of offline and online offers. Yumurtacı Hüseyinoğlu et al. (2018) noted that omni-channel capability has three elements, namely channel consistency, cross-channel and social media. Although the definitions of channel integration in the literature are varied, the concepts of omni-channel commerce for retailers are similar. In other words, it is believed that coordinating and integrating the diverse channels can generate synergies that enhance the effectiveness of each channel and contribute to increase the performance of the retailer. Thus, this study adopted the study of Bendoly et al. (2005), which indicated that informational 
integration and physical integration are two important elements of channel integration, as our proposed omni-channel model.

In the present study, XXX Lutz certainly is on the right path by already having managed the shift from a multi- towards a cross-channel retailer and already uses some aspects of an omni-channel strategy, such as the "XXX Lutz Dream Space Planner", or QR-Codes. However, in order to become a true omni-channel retailer and offer clients a genuinely seamless purchasing experience, the firm is advised to a smoother integration of all its five utilized channels, which are namely its physical stores, the firm's catalogues, websites, mobile applications and social media platforms.

\subsection{Information Integration}

Informational integration refers to the diverse channels providing information to one another. Thus, this study proposed six services to improve informational integration. First, by simultaneously introducing QR-Codes for the retailer's entire product assortment across all suitable channels, the shopper's journey could be made more convenient. The difference to the firm's current channel-strategy, in which QR-Codes are only used for selected premium products in XXX Lutz's catalogues is that the entire product assortment should be linked to profound online product information in its physical stores. Consequently, the primarily used channel is the firm's physical store. This measure serves to connect the firm's physical stores more tightly to the firm's online channel. Additionally, the QR-Codes can be easily shown at the firm's social media platforms and in its mobile application ensuring accessibility across all channels.

Second, XXX Lutz's free "Dream Space Planning Service" is already part of its current channel management strategy, but should be emphasized even more and used to get clients showing online interest to the firm's physical store. Thus, the difference to the current strategy is that it shall be emphasized and promoted even more, not only on its website but also on its social media platforms, in catalogues as well as in its mobile applications with the final aim to get more customers to its online planning service.

Third, the launch of an augmented reality application allowing customers to picture XXX Lutz's products in their home is crucial in order for the firm to be able to compete with IKEA who has already proven to be the first mover when it comes to this feature. Although IKEA pursues a low-cost strategy and targets a different customer segment than XXX Lutz, one must keep this in mind since especially XXX Lutz's customers who pay a premium price in exchange for high-quality products are very likely to expect a comparatively better customer experience. Thus, contrary to its current channel strategy, this measure helps the firm to stay competitive by more tightly linking the physical shopping experience with the retailer's mobile application.

Fourth, introducing a customer review function on the firm's social media platforms may enhance the firm's credibility and give potential customers more security for product- and service purchases. The difference to the current channel strategy is that a customer review function on social media is currently unavailable, but it helps the retailer since it increases the likelihood that customers purchase either via the firm's website, via its mobile application or in the physical store.

Fifth, the firm shall further improve its social media presence and promote it in its catalogues in order to strengthen its reputation. Social media is a crucial communication channel that nowadays almost anyone of later generations uses. Consequently, the client is always up to date and can inform himself. This measure does not differ that much to the firm's current channel strategy, but keeping up a good brand reputation has an impact on all of the firm's sales channels, such as its physical store, its online website as well as its mobile applications.

Sixth, XXX Lutz may introduce an online chat section that is available across all online channels and promoted in its catalogues, so that customers can easily contact the firm's personnel regarding any matter of concern. Even if such an online chat is only available 10 hours a day, customers will accept a firm's chat-opening-hours and feel totally cared for. Moreover, this will reduce the phone, e-mail and in-store traffic when it comes to matters that could easily be solved via an online chat. Thus, the difference is that an online chat section is not available in the retailer's current channel management strategy, but it helps customers since they can easily contact an experienced employee from the physical store via the online chat section.

\subsection{Physical Integration}

Physical integration refers to the possibility of shopping across channels. Thus, this study proposed two services to improve physical integration. First, XXX Lutz store environment design - which literally resembles a maze - has to be improved which could be done by launching a section in XXX Lutz application that virtually guides customers through the store. The sidebenefit of such an application section is that sales forces will have to spend less time on guiding customers through the store and can fully focus on their main tasks - consulting customers as well as selling goods and services. Consequently, and contrary to the current channel strategy, this measure helps to more tightly interlink the firm's physical stores with the firm's mobile application, since customers need to use the mobile navigation application, which represents the primarily used channel for providing this service - to enjoy a smooth in-store experience. 
Second, the XXX Lutz Group is highly advised to let go of its other two sub-divisions "Möbelix" and "Mömax", because it confuses customers and causes doubts about the firm's high-quality product credibility. This measure entirely differs from the firm's current philosophy and has no direct effect on any of its channels. However, it would indirectly affect all of XXX Lutz's sales channels, since letting go of its two "low-price" sub-divisions, that are not the firm's core business anyways, allows the retailer to focus all its efforts on its core business, which is: Delivering its well-known premium XXX Lutz products to clients. The primary channel used for this measure are the retailer's physical stores.

Therefore, we may summarize that informational integration includes fully introducing QR-Codes, emphasizing XXX Lutz's Online Dream Space Planner, launching an augmented reality application, introducing a customer review function, improving its social media presence, introducing an online chat, whereas physical integration includes launching a store navigation application and staying focused on offering customers premium products for XXX Lutz. Figure 3 depicts the proposed services on how XXX Lutz can manage to shift from delivering customers a classy cross-channel experience towards flabbergasting them with an astonishing omni-channel adventure.

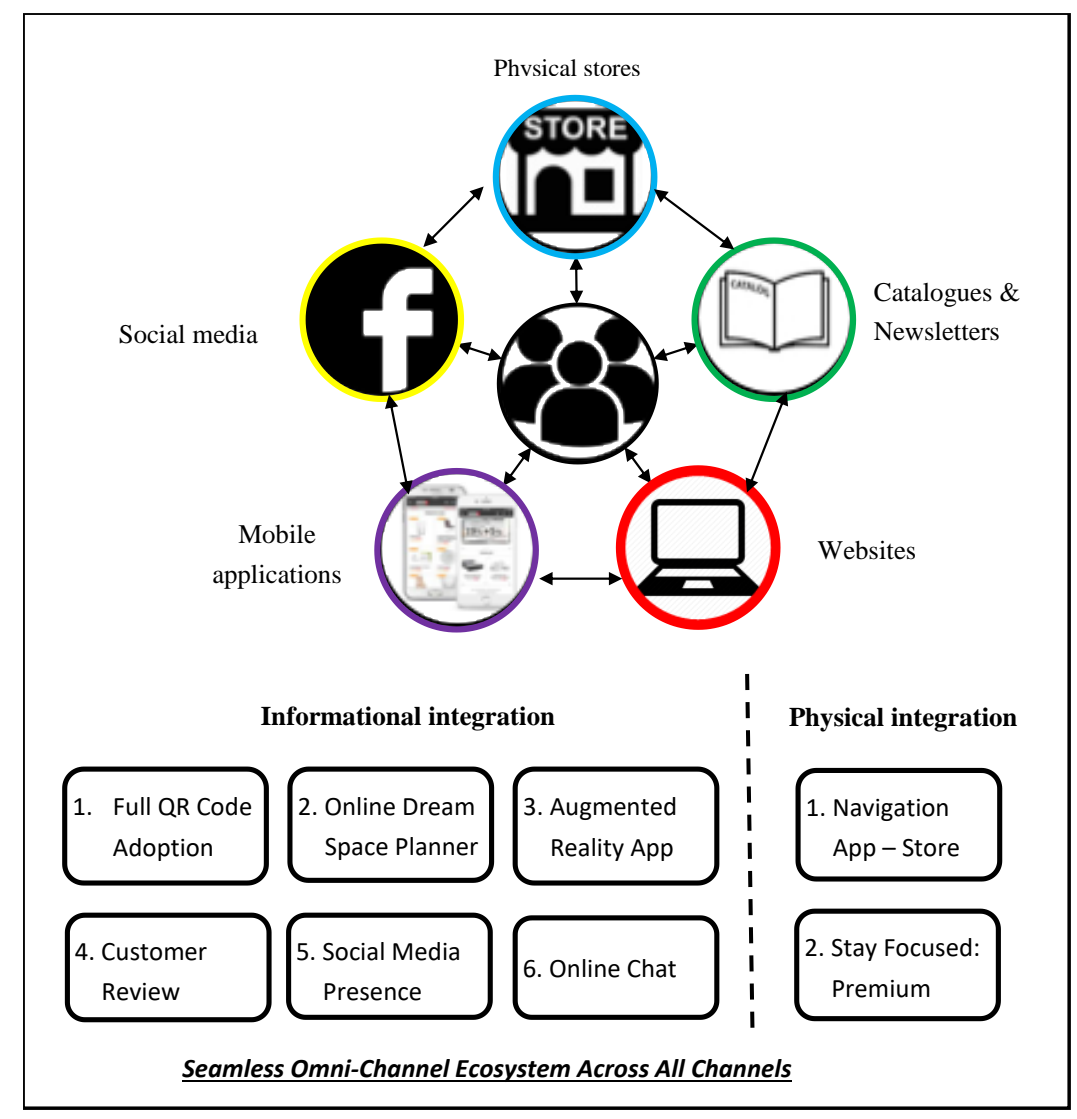

Figure 3. Proposed Omni-Channel services for XXX Lutz

\section{CHALLENGES AND RECOMMENDED SOLUTIONS}

As previously discussed, this study proposed some new services to implement an omni-channel strategy for XXX Lutz. However, shifting to an omni-channel management system may carry ineluctable challenges along. Picot-Coupey et al. (2016) categorized them into strategy-related challenges as well as development-related ones. In the strategy-related challenges, how to keep the consumer within the brand ecosystem and to reinforce lock-in effects (Verhoef et al., 2015). Thus, adding, integrating or blurring channels raises marketing advantages concerning the coordination of the retail mix across channels may cause organizational challenges (Van Baal, 2014). Thus, XXX Lutz is advised to recruit a talented project manager that is capable of identifying and prioritizing challenges that may be coming during the firm's transition. This, in fact, might represent a challenge in itself, because finding a person that has competencies in the fields of both omni-channel retailing, as well as project management, might be tough. Moreover, XXX Lutz omni-channel implementation represents a major change for the firm. As with every organizational change management project, also the culture has to be adapted towards the requirements of the new omni-channel management system. However, managing change properly is not easy. Thus, XXX Lutz is recommended to announce a change management team that supports its newly hired, talented project manager and fosters a culture of change. Additionally, proper omni-channel implementation and culture change must involve the entire 
organization and requires the support of senior managers. Therefore, XXX Lutz is advised to let its senior managers be the first ones officially announcing the new omni-channel strategy, highlighting its importance and communicating it downwards throughout the entire organization.

On the other hand, in the development-related challenges, XXX Lutz may have customer relationship management and information system concerns. For example, potential channel cannibalization might occur between the brick- and click-andmortar store since customers might want to see the actual product but order it online since it appears to be more convenient. Consequently, XXX Lutz's sales forces that are paid on a commission-basis might get very frustrated if they consult a lot of customers, but do not make any sales presuming that most clients order online. This can only be avoided by the implementation of clear rules and regulations. For example, if customers purchase online, but they had been consulted in a store, the sales force should always give clients the personal business card in order for customers to remember their name. The online purchasing process should then contain a section asking the customer if they have been consulted in-store so that they can fill in the sales forces' name, or at least, the store location they had been consulted at. Besides, XXX Lutz must be aware of potential free riding issues (Arora and Sahney, 2018), especially when it comes to its free "Dream Space Planning Service". The firm can overcome this problem by introducing the following limitation: The Dream Space Planning Service, in general, is for free, but the customer does not get any print-outs or electronic files of the plans, unless the client agrees to order something, or pays for the service. This measure ensures that the customer cannot make use of the firm's service unless a purchase is made and thus, free-riding is precluded. Moreover, the new applied technologies - such as the QR-Code, or the Augmented Reality Application - represent major challenges and might even be useless if the customer does not know how to use it. Thus, XXX Lutz is recommended to stick to the study of Lewis et al.'s (2014) advices, who highlight that training of the firm's own staff in the fields of technology and promotion is important. The reason is that the staff's ability to promote used in-store technology represents a good mean to bridge the online and offline experience and educates the customer on how to actually use the available technology. Overall, there may be plenty of other challenges coming up. XXX Lutz may be capable of identifying and prioritizing those unanticipated hurdles for the sake of attaining a holistic omnichannel strategy.

\section{CONCLUSION}

The main objective of this paper is to devise a well-grounded omni-channel commerce strategy for XXX Lutz. Taken together, the proposed services shall be emphasized in order for XXX Lutz to offer customers truly seamless shopping experiences.

The findings suggest that XXX Lutz shift to an omni-channel commerce strategy requires a smoother integration of all utilized channels. This could be achieved by two approaches, namely informational integration and physical integration. Informational integration includes: First, introducing QR-Codes linking the physical product to profound online product information so that the shopper's journey can be made more convenient. Second, XXX Lutz's Online Dream Space Planner shall be emphasized even more and used to get customers showing online interest to the firm's brick-and-mortar store. Third, the launch of an augmented reality application allowing customers to picture XXX Lutz's products in their home is crucial in order for the firm to be able to compete with IKEA. Fourth, introducing a review function for customers may enhance the firm's credibility and get people curious to visit the physical store of such a premium furniture retailer. Fifth, the firm shall further on improve its social media presence in order to strengthen its reputation and keep customers up to date. Sixth, XXX Lutz may introduce an online chat section in which customers can easily contact the firm's personnel regarding any matter of concern. On the other hand, physical integration includes: First, XXX Lutz store environment design - which literally resembles a maze - has to be improved. This could be done by launching a section in XXX Lutz application that virtually guides customers through the store. Second, the XXX Lutz Group is highly advised to let go of its other two lowcost sub-divisions "Möbelix" and "Mömax", because it simply confuses customers and causes them to doubt the firm's highquality product credibility. Moreover, potential challenges XXX Lutz might face by adopting an omni-channel strategy have been identified and adequate solutions proposed.

This study contributes to the academic research by representing a sample guideline on how firms' can design their transition process from a cross-channel towards an omni-channel strategy. Theoretically, this study extends the understanding of the omni-channel strategy for the furniture retailers. Our findings follow the study of Bendoly et al. (2005) and affirm the assumption that informational and physical integrations are two important dimensions of channel integration for the omnichannel retailers. Therefore, this study advances the extant research of channel management in the omni-channel retail context. Practically, we further infer our findings that designing such a guideline for any other firm requires a profound analysis of the selected firm and its sales channels as well as an omni-channel design that corresponds to the firm's needs. The Austrian furniture retailer XXX Lutz was analyzed in the present study. However, the findings of the study cannot be generalized because the sample was restricted to a specific service sector. Although this study clearly has some limitations, we believe that this contribution represents a feasible starting point for XXX Lutz's transition from a cross-channel towards a successful omni-channel retailer. 


\section{REFERENCES}

Arora, S., \& Sahney, S. (2018). Consumer's webrooming conduct: an explanation using the theory of planned behavior. Asia Pacific Journal of Marketing and Logistics, 30(4), 1040-1063.

Beck, N., \& Rygl, D. (2015). Categorization of multiple channel retailing in Multi-, Cross-, and Omni-Channel Retailing for retailers and retailing. Journal of Retailing and Consumer Services, 27, 170-178.

Bendoly, E., Blocher, J. D., Bretthauer, K. M., Krishnan, S., \& Venkataramanan, M. A. (2005). Online/in-store integration and customer retention. Journal of Service Research, 7(4), 313-327.

Buzzvalue. (2018). Möbelhandel im Social Web: XXXLutz und IKEA top. Retrieved May 20, 2019, from BuzzValue.at I Social Media Marktforscher | Wien website: https://www.buzzvalue.at/single-post/2018/04/04/M\%C3\%B6belhandel-im-Social-Web-XXXLutz-und-IKEAtop

Cochoy, F. (Ed.). (2012). The pencil, the trolley, and the smartphone: understanding the future of self-service retailing through its sociotechnical history. In Nordic retail research: emerging diversity (pp. 215-234). Göteborg: BAS.

Frasquet, M., \& Miquel, M. J. (2017). Do channel integration efforts pay-off in terms of online and offline customer loyalty? International Journal of Retail \& Distribution Management, 45(7/8), 859-873.

Gist. (2019). Multi-channel, Cross-channel, Omni-channel: What difference? Retrieved May 18, 2019, from Gist website: https://getgist.com/multi-channel-cross-channel-omni-channel/

Lee, H. H., \& Kim, J. (2010). Investigating dimensionality of multichannel retailer's cross-channel integration practices and effectiveness: shopping orientation and loyalty intention. Journal of Marketing Channels, 17(4), 281-312.

Lewis, J., Whysall, P., \& Foster, C. (2014). Drivers and Technology-Related Obstacles in Moving to Multichannel Retailing. International Journal of Electronic Commerce, 18(4), 43-68. https://doi.org/10.2753/JEC1086-4415180402

Neslin, S. A., Grewal, D., Leghorn, R., Shankar, V., Teerling, M. L., Thomas, J. S., \& Verhoef, P. C. (2006). Challenges and Opportunities in Multichannel Customer Management. In Journal of Service Research (Vol. 9, pp. 95-112). Retrieved from http://journals.sagepub.com/doi/10.1177/1094670506293559

Picot-Coupey, K., Huré, E., \& Piveteau, L. (2016). Channel design to enrich customers' shopping experiences: Synchronizing clicks with bricks in an omni-channel perspective - the Direct Optic case. International Journal of Retail \& Distribution Management, 44(3), 336-368. https://doi.org/10.1108/IJRDM-04-2015-0056

Rigby, D. K. (2011). The Future of Shopping. Harvard Business Review, (December 2011). Retrieved from https://hbr.org/2011/12/the-futureof-shopping

Salma, A., \& Anil, K. (2015). Opportunities and Challenges of Omni-Channel Retailing in the Emerging Market. Journal of Retail Management and Research, 1(1), 1-16.

Schmieder, U.-M. (2010). Integrierte Multichannel-Kommunikation im Einzelhandel (1. Aufl). Wiesbaden: Gabler.

Van Baal, S. (2014). Should retailers harmonize marketing variables across their distribution channels? An investigation of cross-channel effects in multi-channel retailing. Journal of Retailing and Consumer Services, 21(6), 1038-1046.

Verhoef, P. C., Kannan, P. K., \& Inman, J. J. (2015). From multi-channel retailing to omni-channel retailing: introduction to the special issue on multi-channel retailing. Journal of retailing, 91(2), 174-181.

XXX Lutz Group. (2019). Welcome to XXXLUTZ Group. Retrieved May 20, from https://xxxlgroup.com/en/xxx/utz-group.

XXX Lutz. (2019). Möbel bequem online kaufen bei XXXLutz! XXXLutz. Retrieved May 20, from https://www.xxxlutz.at/c/moebel.

Yumurtacı Hüseyinoğlu, I. Ö., Sorkun, M. F., \& Börühan, G. (2018). Revealing the impact of operational logistics service quality on omnichannel capability. Asia Pacific Journal of Marketing and Logistics, 30(5), 1200-1221. 